\title{
A new approach for solving optimal nonlinear control problems using decriminalization and rationalized Haar functions
}

\author{
Zhenyu Han ${ }^{\text {a }}$, Shurong $\mathrm{Li}^{\mathrm{b}}$ \\ College of Information and Control Engineering, China University of Petroleum, \\ Dongying, Shandong Province, China 257061 \\ aemail:hanzhenyu0315@gmail.com \\ bemail (corresponding author):lishuron@upc.edu.cn
}

Keywords: Rationalized Haar functions; Optimal control; Quadratic programming; Inequality constraints.

\begin{abstract}
This paper presents a numerical method based on quasilinearization and rationalized Haar functions for solving nonlinear optimal control problems including terminal state constraints, state and control inequality constraints. The optimal control problem is converted into a sequence of quadratic programming problems. The rationalized Haar functions with unknown coefficients are used to approximate the control variables and the derivative of the state variables. By adding artificial controls, the number of state and control variables is equal. Then the quasilinearization method is used to change the nonlinear optimal control problems with a sequence of constrained linear-quadratic optimal control problems. To show the effectiveness of the proposed method, the simulation results of two constrained nonlinear optimal control problems are presented.
\end{abstract}

\section{Introduction}

A widely used method to solve optimal control problems is the direct method. The direct method converts the optimal control problem into a mathematical programming problem with a large number of parameters and equality constraints [1-4]. In order to solve these problems, Hussein Jaddu [5] proposed a method to solve the linear-quadratic and the nonlinear optimal control problems by using Chebyshev polynomials to approximate the state variables. Also shifted Legendre polynomials to parameterize the derivative of each of the state variables are introduced to solve the linear-quadratic optimal control problem in [6].

In this paper we present a computational method for solving nonlinear optimal control problems with state and control inequality. The quasilinearization technique is applied to convert the constrained optimal control problems into a sequence of constrained linear-quadratic optimal control problems. Then by applying the Haar wavelets functions to approximate the control variables and the derivative of the state variables, the constrained linear-quadratic optimal control problems are transformed into quadratic programming problems and are well solved.

\section{Review of Some Properties of Haar Wavelets}

\subsection{Haar functions}

The Haar function $H_{r}(t), r=1,2,3, \ldots$ are composed of three values $+1,-1$ and 0 can be defined on the interval $[0,1)$ as $[7]$

$$
H_{r}(t)= \begin{cases}1, & J_{1} \leqslant t<J_{1 / 2}, \\ -1, & J_{1 / 2} \leqslant t<J_{0}, \quad J_{u}=(j-u) / 2^{i}, u=0,0.5,1 . \\ 0, & \text { otherwise, }\end{cases}
$$

The value of $r$ defines two parameters $i$ and $j$ via $r=2^{i}+j-1, i=0,1,2,3, \ldots, j=1,2,3, \ldots, 2^{i}$.

$H_{0}(t)$ is defined for $i=j=0$ and is given by $H_{0}(t)=1,0 \leqslant t<1$. 
The orthogonality property is given by

$$
\int_{0}^{1} H_{r}(t) H_{v}(t) d t= \begin{cases}2^{-i}, & r=v \\ 0, & r \neq v\end{cases}
$$

where $v=2^{n}+m-1, n=0,1,2,3, \ldots, m=1,2,3, \ldots, 2^{n}$.

\subsection{Function approximation}

A function $f(t) \in L^{2}[0,1]$, may be expanded into Haar functions as

$$
f(t)=\sum_{r=0}^{\infty} w_{r} H_{r}(t)
$$

where $w_{r}$ are given by $w_{r}=2^{i} \int_{0}^{1} f(t) H_{r}(t) d t, r=0,1,2, \ldots$, with $r=2^{i}+j-1, i=0,1,2,3, \ldots, 2^{i}$, and $r=0$ for $i=j=0$. The series in Eq.(3) contains infinite terms. If, we let $i=0,1,2,3, \ldots, \alpha$ then the infinite series in Eq.(3) is truncated up to its first $s$ terms as

$$
f(t) \approx \sum_{r=0}^{s-1} \alpha_{r} H_{r}(t)=W^{T} \phi(t), \quad s=2^{\alpha+1}, \alpha=0,1,2,3, \ldots
$$

The Haar wavelet functions coefficient vector $w$ and Haar wavelet functions vector $\phi(t)$ are defined as

$$
\begin{aligned}
& W=\left[\alpha_{0}, \alpha_{1}, \ldots, \alpha_{s-1}\right]^{T}, \\
& \phi(t)=\left[\phi_{0}(t), \phi_{1}(t), \ldots, \phi_{s-1}(t)\right]^{T}, \\
& \phi_{r}(t)=H_{r}(t), r=0,1,2, \ldots, s-1 .
\end{aligned}
$$

In Eq.(5) the row denotes the order of the Haar functions. The matrix $\hat{\phi}_{k \times k}$ can be expressed as

$$
\hat{\phi}_{s \times s}=[\phi(1 / 2 s), \phi(3 / 2 s), \ldots, \phi((2 s-1) / 2 s)] .
$$

By using Eqs.(4) and (8) we get

$$
\begin{gathered}
{[f(1 / 2 s), f(3 / 2 s), \ldots, f((2 s-1) / 2 s)]=W^{T} \hat{\phi}_{s \times s} .} \\
W^{T}=[f(1 / 2 s), f(3 / 2 s), \ldots, f((2 s-1) / 2 s)] \hat{\phi}_{s \times s}^{-1} .
\end{gathered}
$$

\subsection{Integration of Haar wavelets}

The integration of the $\phi(t)$ is given by

$$
\int_{0}^{t} \phi\left(t^{\prime}\right) d t^{\prime} \simeq P \phi(t)
$$

where $P=P_{s \times s}$ is the $s \times s$ is the $s \times s$ operational matrix for integration and is given in [9] as

$$
P_{s \times s}=\frac{1}{2 s}\left[\begin{array}{cc}
2 s P_{s / 2 \times s / 2} & -\hat{\phi}_{s / 2 \times s / 2} \\
\hat{\phi}_{s / 2 \times s / 2}^{-1} & 0
\end{array}\right],
$$

with $\hat{\phi}_{1 \times 1}=[1]$ and $P_{1 \times 1}=1 / 2$ respectively. 


\section{Problem statement}

The problem we are considering is to find the control vector $u(\tau)$, and the corresponding state vector $x(\tau), \tau \in\left[0, t_{f}\right]$, which minimize (or maximize) the functional

$$
J=\int_{0}^{t_{f}}\left(x(t)^{T} Q x(t)+u(t)^{T} R u(t)\right) d t
$$

subject to

$$
\begin{aligned}
& \dot{x}(\tau)=f(x(\tau), u(\tau), \tau), \tau \in\left[0, t_{f}\right], \quad x(0)=x_{0}, \\
& \Psi\left(x\left(t_{f}\right), \tau\right)=0 \\
& u(\tau) \leqslant u_{\max }, u(\tau) \geqslant u_{\min }, \\
& x(\tau) \leqslant x_{\max }, x(\tau) \geqslant x_{\min },
\end{aligned}
$$

where $x(\tau) \in R^{n}$ and $u(\tau) \in R^{m}$, are state vector and control vector, respectively. $x_{0}$ is the initial condition vector. $Q$ is a positive semi definite matrix, $R$ is a positive definite matrix. The vector relation $u(\tau) \leqslant u_{\max }$ means that a coordinate $u_{i}(\tau) \leqslant u_{\max , i}$. Also, $t_{f}$ denotes the final time. The vector function $f$ is generally nonlinear and assumed to be smooth with respect to $x$ and $u$. It is assumed that the problem (13)-(18) has a unique solution.

\section{Quasilinearization}

Then the optimal control problem (13)-(17) can be replaced with the following sequence of constrained linear-quadratic optimal control problems

Minimize

$$
J^{k+1}=\int_{0}^{t_{f}}\left(x^{k+1}(\tau)^{T} Q x^{k+1}(\tau)+u^{k+1}(\tau)^{T} R u^{k+1}(\tau)\right) d \tau
$$

subject to

$$
\begin{aligned}
& \dot{x}^{k+1}=A^{k}(\tau) x^{k+1}+B^{k}(\tau) U^{k+1}+h^{k}(\tau), x^{k+1}(0)=x_{0}, \\
& \Psi_{x}\left(x^{k}\left(t_{f}\right), t_{f}\right)\left(x^{k+1}\left(t_{f}\right)-x^{k}\left(t_{f}\right)\right)+\Psi\left(x^{k}\left(t_{f}\right), t_{f}\right)=0, \\
& u^{k+1}(\tau) \leqslant u_{\max }, u^{k+1}(\tau) \geqslant u_{\min }, \\
& x^{k+1}(\tau) \leqslant x_{\max }, x^{k+1}(\tau) \geqslant x_{\min },
\end{aligned}
$$

where

$$
\begin{aligned}
& h^{k}(\tau)=f\left(x^{k}, U^{k}, \tau\right)-A^{k}(\tau) x^{k}-B^{k}(\tau) u^{k}, \\
& A^{k}(\tau)=\partial F(x, u, \tau) /\left.\partial x\right|_{x^{k}, u^{k}}, \\
& B^{k}(\tau)=\partial F(x, u, \tau) /\left.\partial u\right|_{x^{k}, u^{k}} .
\end{aligned}
$$


we assume that $m=n$ and $B^{k}(k)$ is invertible for all $t \in\left[0, t_{f}\right]$. When the assumption is not satisfying and $m<n$, an artificial control vector $z(\tau)$ of dimension $(n-m) \times 1$ is added to modify $B^{k}(\tau)$. Then, the $B^{k}(\tau)$ is changed into $B_{\text {new }}^{k}(t)$ as follow

$$
B_{n e w}^{k}(\tau)=\left[\begin{array}{cc}
I_{n-m} & \\
O_{m \times(n-m)} & B^{k}(\tau)
\end{array}\right],
$$

where $I_{n-m}$ is $(n-m) \times(n-m)$ identity matrix, $O_{m \times(n-m)}$ is a zero matrix. Correspondingly, the control vector $u^{k+1}(\tau)$ is modified to $u_{\text {new }}^{k+1}(\tau)$ as follow

$$
u_{\text {new }}^{k+1}(\tau)=\left[\begin{array}{c}
z(\tau) \\
u^{k+1}(\tau)
\end{array}\right]
$$

and the performance index is modified to

$$
\hat{J}^{k+1}=J^{k+1}+\int_{0}^{t_{f}} z^{T} L z d \tau \text {. }
$$

\section{Problem reformulation}

The time transformation $\tau=t_{f} t$ is introduced in order to use Haar functions defined on $t \in[0,1]$. Using this transformation and expressing the optimal control problem (18)-(22) in terms of $t$, we get Minimize

$$
J^{k+1}=t_{f} \int_{0}^{1}\left(x^{k+1 T} Q x^{k+1}+u^{k+1 T} R u^{k+1}\right) d t
$$

subject to

$$
\begin{aligned}
& \left(t_{f}\right)^{-1} d x^{k+1} / d t=A^{k}(t) x^{k+1}+B^{k}(t) u^{k+1}+h^{k}(t), \quad x^{k+1}(0)=x_{0}, \\
& \Psi_{x}\left(x^{k}(1,1)\right)\left(x^{k+1}-x^{k}(1)\right)+\Psi\left(x^{k}(1), 1\right)=0, \\
& u^{k+1}(t) \leqslant u_{\max }, u^{k+1}(t) \geqslant u_{\text {min }}, \\
& x^{k+1}(t) \leqslant x_{\max }, x^{k+1}(t) \geqslant x_{\text {min }} .
\end{aligned}
$$

Approximating each of the system dynamic functions and control variables by Haar series with unknown parameters gives

$$
\begin{aligned}
& \dot{x}^{k+1}(t)=\hat{\phi}^{T}(t) M, \\
& \hat{\phi}(t)=I \otimes \phi(t), \\
& M=\left[M_{1}^{T}, M_{2}^{T}, \cdots, M_{n}^{T}\right],
\end{aligned}
$$

where $I$ is a $n \times n$ dimensional identity matrix, $\phi(t)$ is $s \times 1$ vector $\left(s=2^{\alpha+1}, \alpha=0,1,2, \cdots\right), \hat{\phi}$ is a matrix of order $n s \times n, M$ is a $n s \times 1$ dimensional vector, and $\otimes$ denotes Kronecker product. $\phi(t)$ is a Haar functions vector which is defined as

$$
\phi(t)=\left[\phi_{0}(t), \phi_{1}(t), \cdots, \phi_{s-1}(t)\right]
$$


Also we have

$$
x(0)=\hat{\phi}(t) d
$$

where $d=\left[d_{1}^{T}, d_{2}^{T}, \cdots, d_{n}^{T}\right]^{T}$. is a vector of order .

Using the Haar functions' integration operational matrix $P, x^{k+1}$ can be determined

$$
x^{k+1}(t)-x(0)=\int_{0}^{t_{f}} \hat{\phi}^{T}\left(t^{\prime}\right) M d t^{\prime}=\left(I \otimes \phi^{T}(t)\right)\left(I \otimes P^{T}\right) M=\hat{\phi}^{T} \hat{P}^{T} M .
$$

where $P$ is an operational matrix of integration given in Eq.(12).

From Eqs.(39) and (43) we obtain

$$
x^{k+1}(t)=\hat{\phi}^{T}(t)\left(d+\hat{P}^{T} M\right)=(I \otimes \phi)^{T}\left(d+\hat{P}^{T} M\right)=\left(I \otimes \phi^{T}\right)\left(d+\hat{P}^{T} M\right),
$$

From Eqs.(32),(40) and (41), the control variables $u^{k+1}$ can be determined as a function of the unknown parameters of the state variables as follows

$$
\begin{aligned}
u^{k+1} & =\left(B^{k}\right)^{-1}\left[\left(t_{f}\right)^{-1} \hat{\phi}^{T} M-A^{k} \hat{\phi}^{T}\left(d+\hat{P}^{T} M\right)-h^{k}\right] \\
& =\left(B^{k}\right)^{-1}\left[\left(t_{f}\right)^{-1}\left(I \otimes \phi^{T}\right) M-A^{k}\left(I_{x} \otimes \phi^{T}\right)\left(d+\hat{P}^{T} M\right)-h^{k}\right]
\end{aligned}
$$

The performance index can be approximated by substituting Eqs.(41) and (42) into Eq.(28) to get

$$
\begin{aligned}
J_{N}^{(k+1)} & =t_{f} \int_{0}^{1}\left[\left(d+\hat{P}^{T} M\right)^{T}(I \otimes \phi) Q\left(I \otimes \phi^{T}\right)\left(d+\hat{P}^{T} M\right) n\right. \\
& +\left(\left(t_{f}\right)^{-1} M^{T}\left(I_{x} \otimes \phi\right)-\left(d+\hat{P}^{T} M\right)^{T}(I \otimes \phi)\left(A^{k}\right)^{T}-\left(h^{k}\right)^{T}\right) F(t) \\
& \left.\times\left(\left(t_{f}\right)^{-1}\left(I \otimes \phi^{T}\right) M-A^{k}\left(I \otimes \phi^{T}\right)\left(d+\hat{P}^{T} M\right)-h^{k}\right)\right],
\end{aligned}
$$

where $J_{N}^{k+1}$ is the approximate value of $J^{k+1}, F(t)=\left(\left(\left(B^{k}\right)^{-1}\right)^{T} R\left(B^{k}\right)^{-1}\right)$. Eq.(44) can be simplified to

$$
\begin{aligned}
& J_{N}^{k+1}=t_{f} \int_{0}^{1}\left[\left(d+\hat{P}^{T} M\right)^{T}\left(Q \otimes \phi \phi^{T}\right)\left(d+\hat{P}^{T} M\right)+\left(t_{f}\right)^{-2} M^{T}\left(F(t) \otimes \phi \phi^{T}\right) M\right. \\
& -\left(t_{f}\right)^{-1} M^{T}\left(F(t) h^{k}(t) \otimes \phi\right)-\left(t_{f}\right)^{-1} M^{T}\left(F(t) A^{k}(t) \otimes \phi \phi^{T}\right)\left(d+\hat{P}^{T} M\right) n \\
& -\left(t_{f}\right)^{-1}\left(d+\hat{P}^{T} M\right)^{T}\left(\left(A^{k}(t)\right)^{T} F(t) \otimes \phi \phi^{T}\right) M n+\left(d+\hat{P}^{T} M\right)^{T}\left(\left(A^{k}(t)\right)^{T} F(t) h^{k}(t) \otimes \phi\right) \\
& -\left(t_{f}\right)^{-1}\left(\left(h^{k}(t)\right)^{T} F(t) \otimes \phi^{T}\right) M n+\left(d+\hat{P}^{T} M\right)^{T}\left(\left(A^{k}(t)\right)^{T} F(t) A^{k}(t) \otimes \phi \phi^{T}\right)\left(d+\hat{P}^{T} M\right) n \\
& \left.+\left(\left(h^{k}(t)\right)^{T} F(t) A^{k}(t) \otimes \phi^{T}\right)\left(d+\hat{P}^{T} M\right)+\left(h^{k}(t)\right)^{T} F(t) h^{k}(t)\right] d t,
\end{aligned}
$$

which can be rewritten as

$$
J_{N}^{k+1}=\frac{1}{2} M^{T} C_{1} M+C_{2}^{T} M+C_{3},
$$


where

$$
\begin{aligned}
C_{1}= & t_{f} \int_{0}^{1}\left[2 \hat{P}\left(Q \otimes \phi \phi^{T}\right) \hat{P}^{T}+2\left(t_{f}\right)^{-2}\left(F(t) \otimes \phi \phi^{T}\right)-2\left(t_{f}\right)^{-1}\left(F(t) A^{k}(t) \otimes \phi \phi^{T}\right) \hat{P}^{T}\right. \\
& \left.-2\left(t_{f}\right)^{-1} \hat{P}\left(\left(A^{k}(t)\right)^{T} F(t) \otimes \phi \phi^{T}\right)+2 \hat{P}\left(\left(A^{k}(t)\right)^{T} F(t) A^{k}(t) \otimes \phi \phi^{T}\right) \hat{P}^{T}\right] d t \\
C_{2}= & t_{f} \int_{0}^{1}\left[2 d^{T}\left(Q \otimes \phi \phi^{T}\right) \hat{P}^{T}-2\left(t_{f}\right)^{-1} d^{T}\left(\left(A^{k}(t)\right)^{T} F(t) \otimes \phi \phi^{T}\right)+2 d^{T}\left(\left(A^{k}(t)\right)^{T} F(t) A^{k}(t) \otimes \phi \phi^{T}\right) \hat{P}^{T}\right. \\
& \left.-2\left(t_{f}\right)^{-1}\left(\left(h^{k}\right)^{T} F(t) \otimes \phi^{T}\right)+2\left(\left(h^{k}\right)^{T} F(t) A^{k}(t) \otimes \phi^{T}\right) \hat{P}^{T}\right] d t \\
C_{3} & =t_{f} \int_{0}^{1}\left[d^{T}\left(Q \otimes \phi \phi^{T}\right) d+d^{T}\left(\left(A^{k}(t)\right)^{T} F(t) A^{k}(t) \otimes \phi \phi^{T}\right) d\right. \\
& \left.+d^{T}\left(\left(A^{k}(t)\right)^{T} F(t) h^{k}(t) \otimes \phi\right)+\left(\left(h^{k}\right)^{T} F(t) A^{k}(t) \otimes \phi^{T}\right) d+\left(h^{k}\right)^{T} F(t) h^{k}\right] d t
\end{aligned}
$$

The terminal equality state constraints can be approximated by substituting Eq.(41) into Eq.(31) as follows

$$
\psi_{x}\left(x^{k}(1), 1\right)\left(I_{x} \otimes \phi^{T}\right) \hat{P}^{T} M=-\psi_{x}\left(x^{k}(1), 1\right)\left(\left(I_{x} \otimes \phi^{T}\right) d-x^{k}(1)\right)-\psi\left(x^{k}(1), 1\right),
$$

Then we approximate the inequality constraints of the optimal problem. These constraints can be handled by requiting their satisfaction at a finite number of discrete points, $0=t_{0}<t_{1}<\cdots<t_{N-1}=1$. By substituting Eqs.(41) and (42) into Eqs.(34) and (33) respectively, we get

$$
\begin{aligned}
& \quad\left(B^{k}\left(t_{i}\right)\right)^{-1}\left[\left(t_{f}\right)^{-1}\left(I \otimes \phi^{T}\left(t_{i}\right)\right)-A^{k}\left(I \otimes \phi^{T}\left(t_{i}\right)\right) \hat{P}^{T}\right] M \leqslant\left(B^{k}\left(t_{i}\right)\right)^{-1}\left[A^{k}\left(I \otimes \phi^{T}\left(t_{i}\right)\right) d+h^{k}\left(t_{i}\right)\right]+U_{\max }, \\
& -\left(B^{k}\left(t_{i}\right)\right)^{-1}\left[\left(t_{f}\right)^{-1}\left(I \otimes \phi^{T}\left(t_{i}\right)\right)-A^{k}\left(I \otimes \phi^{T}\left(t_{i}\right)\right) \hat{P}^{T}\right] M \leqslant-\left(B^{k}\left(t_{i}\right)\right)^{-1}\left[A^{k}\left(I \otimes \phi^{T}\left(t_{i}\right)\right) d+h^{k}\left(t_{i}\right)\right]-U_{\min }, \\
& \quad\left(I \otimes \phi^{T}\left(t_{i}\right)\right) \hat{P}^{T} M \leqslant-\left(I \otimes \phi^{T}\left(t_{i}\right)\right) d+X_{\max }, \\
& -\left(I \otimes \phi^{T}\left(t_{i}\right)\right) \hat{P}^{T} M \leqslant\left(I \otimes \phi^{T}\left(t_{i}\right)\right) d-X_{\text {min }} .
\end{aligned}
$$

From the previous reformulation, the optimal control problem (28)-(33) can be approximated by the following quadratic programming problem

$$
\min _{M} \frac{1}{2} M^{T} C_{1} M+C_{2} M+C_{3}
$$

subject to

$$
\begin{aligned}
& F_{1} M-b_{1}=0, \\
& F_{2} M-b_{2} \leqslant 0 .
\end{aligned}
$$

where

$$
\begin{aligned}
& F_{1}=\psi_{x}\left(x^{k}(1), 1\right)\left(I \otimes \phi^{T}\right) \hat{P}^{T}, \\
& b_{1}=-\psi_{x}\left(x^{k}(1), 1\right)\left(\left(I \otimes \phi^{T}\right) d-x^{k}(1)\right)-\psi\left(x^{k}(1), 1\right), \\
& F_{2}=\left[\begin{array}{l}
\left.\left.+\left(B^{k}(t)\right)\right)^{-1}\left[\frac{1}{t_{f}}\left(I \otimes \phi^{T}(t)\right)\right)-A^{k}\left(I \otimes \phi^{T}(t)\right) \hat{P}^{T}\right] \\
\left.\left.\left.-\left(B^{k}(t)\right)\right)^{-1}\left[\frac{1}{t_{f}}\left(I \otimes \phi^{T}(t)\right)\right)-A^{k}\left(I \otimes \phi^{T}(t)\right)\right) \hat{P}^{T}\right] \\
\left.+\left(I \otimes \phi^{T}(t)\right)\right) \hat{P}^{T} \\
\left.-\left(I \otimes \phi^{T}(t)\right)\right) \hat{P}^{T}
\end{array}\right],
\end{aligned}
$$




$$
b_{2}=\left[\begin{array}{l}
\left.\left.+\left(B^{k}(t)\right)\right)^{-1}\left[A^{k}\left(I \otimes \phi^{T}(t)\right)\right) d+h^{k}\left(t_{i}\right)\right]+U_{\max } \\
\left.\left.\left.-\left(B^{k}(t)\right)\right)^{-1}\left[A^{k}\left(I \otimes \phi^{T}(t)\right)\right) d+h^{k}(t)\right)\right]-U_{\min } \\
\left.-\left(I \otimes \phi^{T}(t)\right)\right) d+X_{\max } \\
\left.+\left(I \otimes \phi^{T}(t)\right)\right) d-X_{\min }
\end{array}\right] .
$$

Problem (49)-(55) is a standard quadratic programming problem.

After obtaining the optimal solution of the unknown parameters $M$, we substitute these parameters into (41) and (42) to obtain the new nominal states $x^{k}(t)$ and nominal control $u^{k}(t)$ to be used in the next iteration. These new nominal trajectories have to be substituted in Eqs.(28)-(33) to get the next constrained linear quadratic optimal control problem. This procedure has to be repeated until an acceptable convergence is achieved. In this paper, the computation is terminated, when the following criterion is satisfied $\left|J_{N}^{k+1}-J_{N}^{k}\right|<\epsilon$, where $\epsilon$ is a sufficiently small required number.

\section{Numerical simulations}

In this section, we consider the Van der Pol oscillator problem. This example is adapted from [5] and studied by using Chebyshev method. Find the control vector $u(t)$ which minimizes and the following terminal state constraints and inequality control constraints

$J=\frac{1}{2} \int_{0}^{5}\left(x_{1}^{2}+x_{2}^{2}+u^{2}\right) d \tau$

subject to

Table 1. Simulation results

\begin{tabular}{ll}
\hline Methods & $\hat{J}^{k+1}$ \\
\hline Quasilinearization and Chebyshev polynomials & \\
$\mathrm{N}=6$ & 2.26330 \\
$\mathrm{~N}=6$ & 2.16015 \\
$\mathrm{~N}=6$ & 2.14208 \\
Present & \\
$\mathrm{k}=16$ & 2.17857 \\
$\mathrm{k}=16$ & 2.15950 \\
$\mathrm{k}=16$ & 2.14959 \\
\hline
\end{tabular}

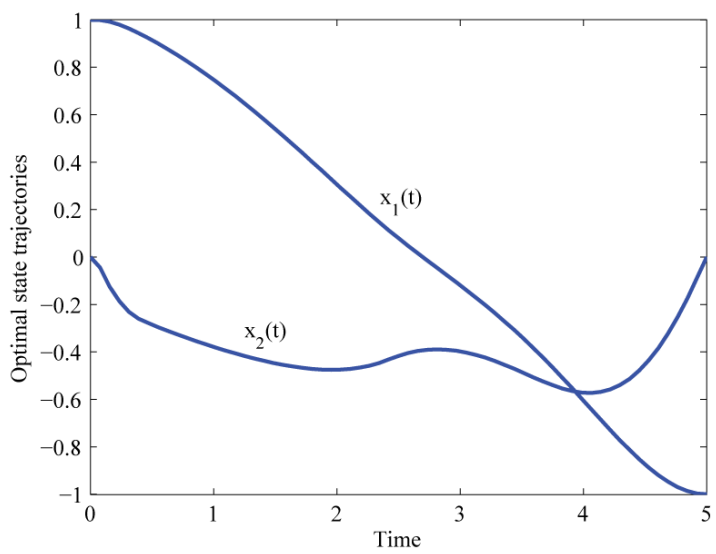

Fig 1. Optimal state trajectories.

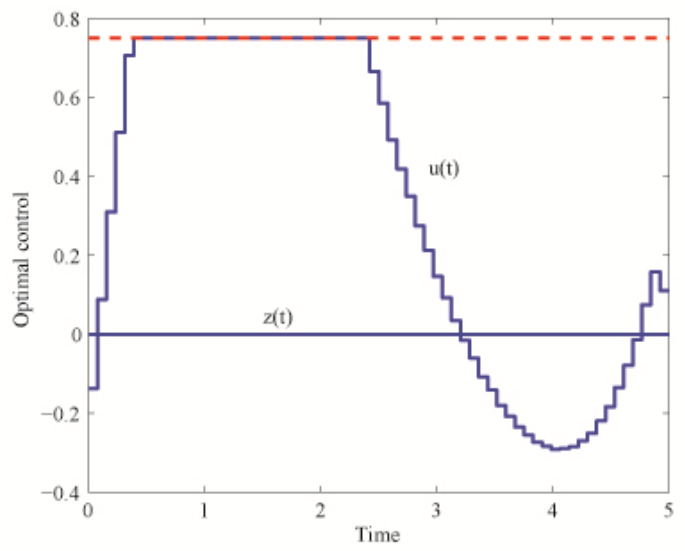

Fig 2. Optimal control. 


$$
\begin{array}{ll}
\dot{x}_{1}=x_{2}, & \dot{x}_{2}=-x_{1}+\left(1-x_{1}^{2}\right) x_{2}+u, \\
\Psi_{1}=x_{1}(5)+1=0, & \Psi_{2}=x_{2}(5)=0, \\
|u(t)| \leqslant 0.75 . &
\end{array}
$$

In Table.1, we compared the optimal solutions obtained using the proposed method with other solutions in the literature. For $k=64$, the computational results for $x_{1}(t), x_{2}(t), u(t)$ and $z(t)$ are given in Figs. 1 and 2, respectively.

\section{Conclusion}

In this paper, we develop an efficient method for solving nonlinear optimal control problems with terminal constraints, state inequality constraints and inequality control constraints. The technique is based on approximation of both the controls and the derivative of the state into Haar series and converting the optimal control problem into a sequence of linear-quadratic optimal control problems. Illustrative examples are included to demonstrate the effectiveness of the proposed method.

\section{References}

[1] C. F. Chen, C. H. Hsiao, A walsh series direct method for solving variational problems, Journal of the Franklin Institute 300 (4) (1975) 265 -280.

[2] M. Razzaghi, S. Yousefi, Legendre wavelets direct method for variational problems, Mathematics and Computers in Simulation 53 (3) (2000) 185- 192.

[3] I.-R. Horng, J.-H. Chou, Shifted chebyshev direct method for solving variational problems, International Journal of Systems Science 16 (7) (1985) 855-861.

[4] M. Razzaghi, M. Razzaghi, A. Arabshahi, Solutions of convolution integral and fredholm integral equations via double flourier series, Applied Mathematics and Computation 40 (3) (1990) $215-224$.

[5] H. Jaddu, Direct solution of nonlinear optimal control problems using quasilinearization and chebyshev polynomials, Journal of the Franklin Institute 339 (4-5) (2002) 479 - 498.

[6] M. Razzaghi, G. N. Elnagar, Linear quadratic optimal control problems via shifted legendre state parameterization, International Journal of Systems Science 25 (1994) 393-399.

[7] M. Razzaghi, Y. Ordokhani, Solution of nonlinear volterra-hammerstein integral equations via rationalized haar functions, Mathematical Problems in Engineering 7 (2) (2001) 205-219.

[8] M. Ohkita, Y. Kobayashi, An application of rationalized haar functions to solution of linear deferential equations 33 (9) (1986) 853-862.

[9] M. Razzaghi, Y. Ordokhani, Solution of deferential equations via rationalized haar functions, Mathematics and Computers in Simulation 56 (3) (2001) 235-246.

[10]V.Yen, M. Nagurka, Optimal control of linearly constrained linear systems via state parameterization, Optimal Control Appl. Mehods 25(1992) 393-399.

[11]D. Kleinman, T. Fortmann, M. Athans, B. Bolt, Newman, On the design of linear systems with piecewise-constant feedback gains, IEEE Trans.Automat. Contr. 13 (1968) 354-361.

[12]J. Vlassenbroeck, A chebyshev polynomial method for optimal control with state constraints, Automatica 24 (4) (1988) 499 - 506.

[13] V. Yen, M. Nagurka, Linear quadratic optimal control via fourier-based state parameterization, Journal of Dynamic Systems, Measurement, and Control 113 (2) (1991) 206-215. 